\title{
Mobile Augmented Reality and Adaptive Art
}

\author{
A game-based Motivation for Energy Saving \\ René Bühling ${ }^{1}$, Mohammad Obaid ${ }^{1,2}$, Stephan Hammer ${ }^{1}$, Elisabeth André ${ }^{1}$ \\ ${ }^{1}$ Human Centered Multimedia, Augsburg University, Germany \\ ${ }^{2}$ Human Interface Technology Lab New Zealand, University of Canterbury, New Zealand \\ \{buehling, mohammad.obaid, hammer, andre\}@informatik.uni-augsburg.de
}

\begin{abstract}
We present the design of an educational treasure hunt game that uses mobile Augmented Reality (AR) and adaptive virtual gardens to raise awareness on energy consumption ways. Within the game, AR is used to present $3 \mathrm{D}$ content that allows for visually understanding the energy consumption problems and their solutions. In addition, the players' performances are given in a form of a visual feedback as dynamic virtual gardens that change from poor to good status. Initial tests show that the presented game is highly appealing to players and motivated them to be aware of the presented problem using AR technologies and the visual effects of their virtual garden's health. Future work will focus on evaluating the learnability and engagement of players.
\end{abstract}

\section{Categories and Subject Descriptors}

[Human-centered computing]: Ubiquitous computing

\section{Keywords}

Energy Awareness, Augmented Reality, Adaptive Art, Dynamic Visualization, Games, Mobile Devices

\section{MOTIVATION}

Electricity consumption is considered to be one of the main factors that increase the amount of Carbon dioxide $\left(\mathrm{CO}_{2}\right)$ emissions into our environment, which is the main substance that causes the global warming effects. In general, a lot of our energy gets wasted and utterly consumed, without care, because we are not aware of the consumption and how it can introduce environmental issues. Therefore, motivated by the future trends of mobile Augmented Reality (AR) [1], we designed a based game scenario that can address the problem of energy consumption and also raise the learnability of possible solutions. In this paper, we propose a novel way of using mobile AR in combination with adaptive virtual gardens to help raise awareness for reducing energy consumption through a game setup. We utilize visual adaption to give feedback on the quality of the individual's environmen-

Copyright is held by the author/owner(s). This is the author's version of the work. It is posted here for your personal use. Not for redistribution. The definitive Version of Record was published in:

MUM '12, Dec 04-06 2012, Ulm, Germany ACM 978-1-4503-1815-0 tal awareness and in reverse to motivate players to reach the most beautiful results.
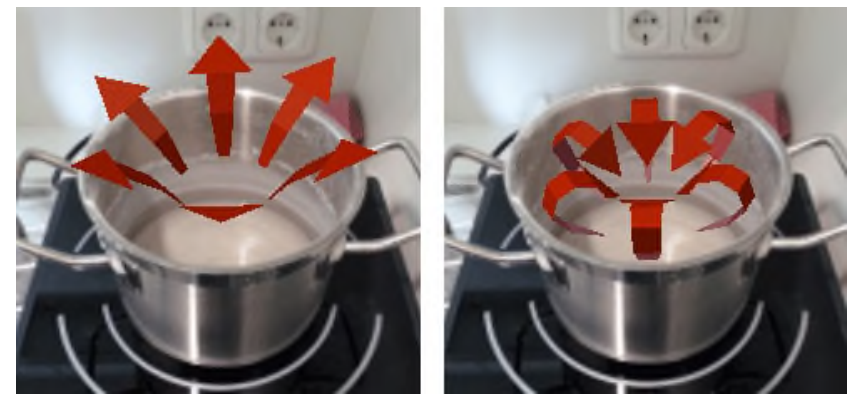

Figure 1: Example of AR content that is used in the game to visualize energetic effects. Left: Loss of heat when cooking with an open pot. Right: Restrained heat when cooking with a capped pot.

\section{GAME DESIGN}

The game is designed in two parts: (1) an $\mathrm{AR}^{1}$ based quiz that confronts players explicitly with environment related issues and (2) a dynamic visualization that emphasizes the impact of the earned players' scores on virtual gardens.

\subsection{AR Treasure Hunt}

Players are grouped into teams and are asked to go through a playful treasure hunt scenario by finding and visiting multiple station points that mark examples of improper energy consumption within a building. At each station, teams are challenged to understand a problem and to solve a quiz question for a better energy consumption practice. In addition, each station has a navigation clue on how to get to the next station in the building. The game content (problem, solution and clues) is presented to players through an AR phone application. Thus, each team is equipped with a mobile phone configured with the AR game application. AR markers are used at each station to mount the graphical content that corresponds to the problem to be solved. Graphical content allows the user to visually understand the issues that relate to the question. Figure 1 illustrates an example of the augmentation presented to users to demonstrate the loss and saving of energy. The explicit visualization and explanation confronts the participants with facts on environmental

\footnotetext{
${ }^{1}$ The AR content was developed on the Android platform using (AndAR): http://code.google.com/p/andar/
} 


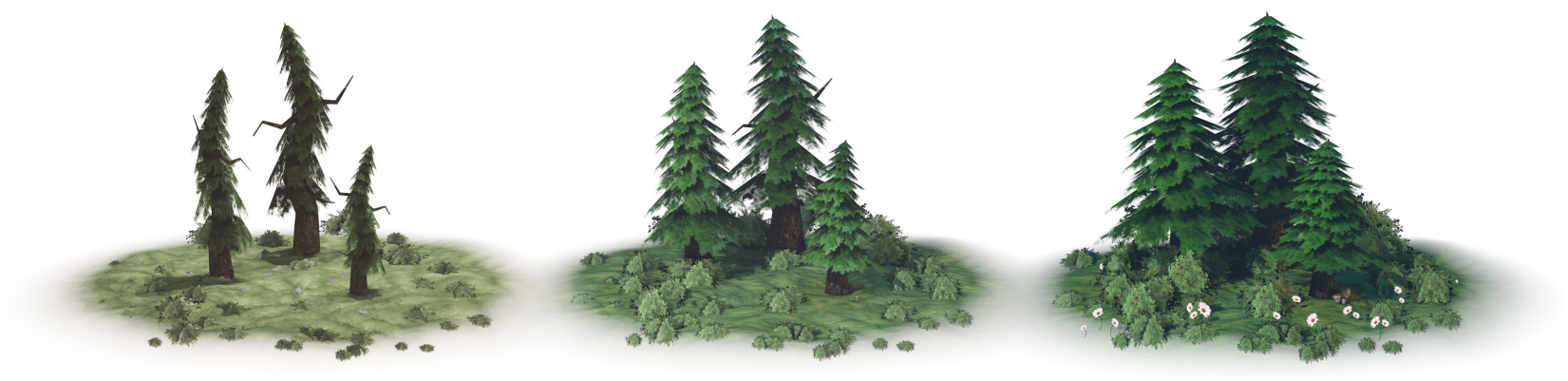

Figure 2: The virtual garden representation that is assigned to each team. From left to right, the 3D representations show a seamless transition from a very weak to a very healthy appearance of the trees.

friendly behaviors and may increase the awareness for the presented topic. AR technologies turn the presented problem into a more lively real world experience, compared to just presenting the user with theoretical information.

\subsection{Score and Competition}

While the AR treasure hunt is mainly related to awareness, a second component is used to increase the player's motivation. As it is a basic principle of gaming to earn any kind of prize, we integrated a visual interpretation of each team's game score. At the start of the game, each team is assigned to be responsible for the health of a personal virtual garden, which is in a very poor health condition at first. The more points players collect at the quiz stations of the game, the better gets the health of the groups' virtual garden. Fig. 2 give an impression on different visualization steps for the health condition of the virtual garden. Using public monitor screens, mounted in several locations in a building, the status of each virtual garden (the team's score) is visible to all competing teams. Two motivation factors raise from this principle: Players are motivated to improve the health of their garden as much as possible. On the other hand, the competitive factor of the public displays raises the motivation to not only have a good, but the best results. Additionally, we designed the theme of gardening and the health of its nature to refer to the topic of environmental protection, which in turn reflects on the concept of the game.

\subsubsection{Adaptive Art in Games}

Graphical real-time environments that are aware of their users and accordingly change to users' behavior have attracted the attention of researchers in recent years, in particular focusing on the different aspects of cinematography such as lighting and camera motion $[3,4,5]$. Moreover, previous work [2] presented how classic artists utilize stylistic methods like composition and shape to emphasize the expression of their work and how such principles can be applied to dynamic and interactive dramaturgy. In our game prototype we used procedural color processing, different light models and levels of asset details, which are adjusted to the intended dramaturgical expression of the garden's health state.

\section{IMPRESSIONS AND FUTURE WORK}

To raise the awareness on energy consumption we created a treasure hunt quiz game where $\mathrm{AR}$ is used to visualize effects of energy waste and savings. To increase the motivation of the player, a global aim was defined by mapping the collected quiz scores to the health of a virtual landscape based on an adaptive virtual garden. Initial game tests were done with teenage girls who played the game in teams of two. It was observed during the game, and was also stated explicitly when asked afterwards, that the participants were highly appealed and motivated by both, the AR quiz tour and the visible effects of their game progress on the health of their virtual gardens. A main limitation noted when using the AR technology is that few players were overwhelmed by the 3D graphical content presented in the real-world, which in turn diverted them from the aim of the game. This factor has to be considered when designing a full scale game and evaluations. Future work is put towards evaluating the presented AR game and investigating the effect of the adaptive virtual gardens on the player's learnability and engagement.

\section{ACKNOWLEDGEMENT}

This research is related to the IT4SE project, funded by BMBF. For more information visit http://www.it4se.net.

\section{REFERENCES}

[1] M. Billinghurst. The Future of Augmented Reality in Our Everyday Life. In Proceedings of the 19th International Display Workshops, Nagoya, Japan, December 2011.

[2] R. Bühling, E. Brihi, M. Wißner, and E. André. Adaptive art - a shape language driven approach to communicate dramaturgy and mood. In M. Si, D. Thue, E. André, J. Lester, J. Tanenbaum, and V. Zammitto, editors, Interactive Storytelling, volume 7069 of Lecture Notes in Computer Science, pages 290-293. Springer Berlin / Heidelberg, 2011.

[3] M. Christie and P. Olivier. Camera control in computer graphics: models, techniques and applications. In $A C M$ SIGGRAPH ASIA 2009 Courses, SIGGRAPH ASIA '09, pages 3:1-3:197, New York, NY, USA, 2009. ACM.

[4] M. S. El-Nasr, J. Zupko, and K. Miron. Intelligent lighting for a better gaming experience. In $\mathrm{CHI}$ '05 extended abstracts on Human factors in computing systems, CHI EA '05, pages 1140-1141, New York, NY, USA, 2005. ACM.

[5] K. Kennedy and R. E. Mercer. Planning animation cinematography and shot structure to communicate theme and mood. In Proceedings of the 2nd international symposium on Smart graphics, SMARTGRAPH '02, pages 1-8, New York, NY, USA, 2002. ACM. 